\title{
Fluid Dynamical Instabilities in Magnetized Partially Ionized Dense Dusty Plasma
}

\author{
Anil Kumar ${ }^{1 *}$, Nagendra Kumar ${ }^{2}$ and Vinod Kumar ${ }^{3}$ \\ ${ }^{1}$ Department of Applied Mathematics, Maharaja Agrasen Institute of Technology, Sec-22, Rohini, Delhi, India \\ ${ }^{2}$ Department of Mathematics, M.M.H. College, Ghaziabad (UP), India \\ ${ }^{3}$ Flat No 304, IInd Floor, Block 20, Omicron III, Greater Noida (UP), India \\ "Corresponding Author: anilkkang@gmail.com, Tel.: +91-8800129272
}

Available online at: www.isroset.org

Received: 05/Dec/2018, Accepted: 13/Dec/2018, Online: 31/Dec/2018

\begin{abstract}
Fluid dynamical instabilities in magnetized partially ionized dense dusty plasma are studied by taking into account relative flow between dust and neutral gas. Following Hurwitz criterion, the onset criteria for instabilities are derived for different densities of the neutral gas and dust components across the interface. It is found that in case of no significant magnetic field stabilization occurs not only due to dust neutral gas collisions but due to relative flow also. Our result might be useful in many situations of astrophysical magnetized dusty plasma namely comets and circumsteller dusty disk e.g. T-Tauri stars.
\end{abstract}

\section{Keywords—Dusty Plasma, Partially Ionized Plasma, Instabilities}

\section{INTRODUCTION}

Instabilities are ubiquitous in partially ionized dusty plasmas. The study of instabilities in partially ionized dusty plasmas has drawn considerable interest in recent past in many different astrophysical contexts such as accretion disks [1] and relativistic jets [2]. The Rayleigh-Taylor instability may be responsible for the formation of waves and bubbles in the Earth's equatorial region [3,4]. D'Angelo [5] studied the Rayleigh-Taylor instability in such a dusty plasma where the dust grains have been assumed to be massive. He investigated the effects of negatively and positively charged dust grains on the gravitational Rayleigh-Taylor instability and found that negatively charged dust have stabilizing effects. A flute like instability which is different from the usual Rayleigh-Taylor instability is investigated by Varma and Shukla [6]. Another important instability, which occurs when adjacent layers of fluid are in relative motion, is called Kelvin-Helmholtz instability and has been analyzed for a conductive magnetized incompressible fluids streaming along the direction of the magnetic field[7].

Goertz [8] described various phenomena and instabilities occurring in dusty plasma of solar system in his review paper. Shear flows play an important role in the dynamics of partially ionized dusty plasma because they induce the unstable KelvinHelmholtz modes in various physical situations namely, superwinds of primeval galaxies in the intergalactic medium [9] and the amplification of self induced magnetic fields in the early Universe [10]. The existence of fluid dynamical instabilities for the partially ionized flow have been discussed by Kamaya and Nishi [11]. They found that the instability of the Alfve'n wave for any $n$ and the two fluid instability for any ' $k$ ' if $n=1$. The Alfven instability appears when its wave number is smaller than a critical value.

Birk [12] derived criteria for unstable Rayleigh-Taylor modes in partially ionized dusty plasma for different density characteristics of the neutral gas and dust components across the interface and found that dust-neutral gas collisions limit the range of unstable wavelengths. Shear flow instabilities in magnetized partially dense dusty plasma have been studied by Birk and Wiechen [13]. They derived onset criteria for instabilities with and without electrical resistivity and found that momentum exchange between the dust and neutral gas stabilized long wavelength perturbations. Excited unstable modes lead to the formation of current sheets and vortices.

In the present paper, we study the fluid dynamical instabilities in magnetized partially ionized dense dusty plasma by taking relative flow between dust and neutral gas. The plan of the paper is as follows. In section 2, the problem is formulated in terms of basic equations governing the motion. Instabilities criteria are obtained and compared with previous studies in section 3 . 


\section{THEORY AND BASIC EQUATIONS}

We consider a magnetized partially ionized dense dusty plasmas whose dynamics are governed by dust and neutral gas components. The dynamics of dusty plasma are characterized by collective behavior for the parameter regime of ordering $a<<d<<\lambda_{d}$, where $a, d$ and $\lambda_{d}$ are the dust grain radius, the average inter-grain distance and the plasma Debye length. The electrons dynamics is not considered as the electrons have no significant influence on the overall behaviuor of dusty plasma. The plasma is considered quasineutral. So we have

$$
n_{i} z_{i}=n_{d} z_{d}+n_{e}
$$

where $n$ and $z$ are number density and charge number and the suffixes $i, d, e$ denote ion, dust and electron fluids respectively. Since the assumption of incompressibility holds very well for the perturbations with velocity amplitudes well below the dustneutral gas sound velocities with propagation time scales larger than sound time scales. The dynamics of fluids are taken incompressible $\left(\nabla \cdot \mathbf{v}_{\mathbf{d}}=\nabla \cdot \mathbf{v}_{\mathbf{n}}=0\right)$. The relevant equations governing the motion of dense dusty plasma are as follows [12]:

$$
\begin{aligned}
& \frac{\partial \rho_{d}}{\partial t}+\left(\mathbf{v}_{\mathbf{d}} \cdot \nabla\right) \rho_{d}=0 \\
& \frac{\partial \rho_{n}}{\partial t}+\left(\mathbf{v}_{\mathbf{n}} \cdot \nabla\right) \rho_{n}=0
\end{aligned}
$$

Here $\rho_{d, n}$ are the dust and neutral gas densities and $\mathbf{v}_{\mathbf{d}, \mathbf{n}}$ are their velocities. The momentum equations for the charged components of the fluid dusty plasma where the ion and electron inertia are negligible in the considered dense dusty plasma, and neutral gas are given by

$$
\begin{gathered}
\rho_{d} \frac{\partial v_{d}}{\partial t}+\left(\mathbf{v}_{\mathbf{d}} \cdot \nabla\right) \mathbf{v}_{\mathbf{d}}=\nabla\left(p_{d}+p_{i}+p_{e}\right)+\frac{1}{4 \pi}(\nabla \times \mathbf{B}) \times \mathbf{B}-\rho_{d} v_{d n}\left(\mathbf{v}_{\mathbf{d}}-\mathbf{v}_{\mathbf{n}}\right)+\rho_{d} \mathbf{g} \\
\rho_{n} \frac{\partial \mathbf{v}_{\mathbf{n}}}{\partial t}+\left(\mathbf{v}_{\mathbf{n}} \cdot \nabla\right) \mathbf{v}_{\mathbf{n}}=-\nabla p_{n}+\rho_{d} v_{d n}\left(\mathbf{v}_{\mathbf{d}}-\mathbf{v}_{\mathbf{n}}\right)+\rho_{n} \mathbf{g}
\end{gathered}
$$

where $p_{d, i, e, n}$ are the dust, ion and neutral gas pressures. The symbols $\mathbf{B}, \mathbf{g}$ and $v_{d n}$ are the magnetic field, the gravitational acceleration and the effective elastic collision frequency between the dust and neutral gas particles respectively. The electron partial pressure is usually negligible in the total pressure of charged components $p_{c}=p_{d}+p_{i}+p_{e}$. The magnetic induction equation is

$$
\frac{\partial \mathbf{B}}{\partial t}=\nabla \times\left(\mathbf{v}_{\mathbf{d}} \times \mathbf{B}\right)
$$

The resistivity, Hall effect as well as other small effect of magnetic field generation are not considered.

We consider equilibrium state where the homogeneous magnetic field $\mathbf{B}_{\mathbf{0}}$ is taken along the $z$-axis and the homogeneous gravitational field $\mathbf{g}=-g \hat{e}_{y}$ We consider interface along the $z$-axis i.e along the equilibrium flow and magnetic field. The other equilibrium quantities on either side of the interface are of the form

$$
\rho_{d 0}=\left\{\begin{array}{l}
\rho_{d I}, y>0, \\
\rho_{d I I}, y<0,
\end{array}\right.
$$




$$
\rho_{n 0}= \begin{cases}\rho_{n I}, & y>0 \\ \rho_{n I I}, & y<0\end{cases}
$$

\section{DERIVATION OF ONSET CRITERIA}

A constant relative velocity of dust to the neutral gas is assumed $\mathbf{V}_{\mathbf{0}}$ with motion $\mathbf{V}_{\mathbf{d} \mathbf{0}}$ in dust and $\mathbf{v}_{\mathbf{n} \mathbf{0}}$ in neutral gas and $\mathbf{v}_{\mathbf{d} 0} \neq \mathbf{v}_{\mathbf{n} 0}$ is taken along the $z$-axis and is such that

$$
\mathbf{v}_{\mathrm{d} 0}-\mathbf{v}_{\mathrm{n} 0}=\mathbf{v}_{\mathbf{0}}
$$

Let $\mathbf{v}^{\prime}, \mathbf{B}^{\prime}, \rho^{\prime}$ and $p^{\prime}$ denote the perturbed quantities for velocity, magnetic field, density and pressure due to a small disturbance to the system. Linearizing equations (1)-(6) about the equilibrium, we obtain

$$
\begin{gathered}
\frac{\partial \rho_{d}^{\prime}}{\partial t}+\left(\mathbf{v}_{\mathbf{d} \mathbf{0}} \cdot \nabla\right) \rho_{d}^{\prime}=0 \\
\frac{\partial \rho_{n}^{\prime}}{\partial t}+\left(\mathbf{v}_{\mathbf{n} \mathbf{0}} \cdot \nabla\right) \rho_{n}^{\prime}=0 \\
\rho_{d 0}\left(\frac{\partial \mathbf{v}_{\mathbf{d}}^{\prime}}{\partial t}+\left(\mathbf{v}_{\mathbf{d} \mathbf{0}} \cdot \nabla\right) \mathbf{v}_{\mathbf{d}}^{\prime}\right)=-\nabla p_{c}^{\prime}+\frac{1}{4 \pi}\left(\nabla \times \mathbf{B}^{\prime}\right) \times \mathbf{B}_{\mathbf{0}}-\rho_{0} v_{d n}\left(\mathbf{v}_{\mathbf{d}}^{\prime}-\mathbf{v}_{\mathbf{n}}^{\prime}\right)+\rho_{d}^{\prime} v_{d n}\left(\mathbf{v}_{\mathbf{d} \mathbf{0}}-\mathbf{v}_{\mathbf{n} \mathbf{0}}\right)+\rho_{d}^{\prime} \mathbf{g} \\
\rho_{n 0}\left(\frac{\partial \mathbf{v}_{\mathbf{n}}^{\prime}}{\partial t}+\left(\mathbf{v}_{\mathbf{n} \mathbf{0}} \cdot \nabla\right) \mathbf{v}_{\mathbf{n}}^{\prime}\right)=-\nabla p_{n}^{\prime}+\rho_{d 0} v_{d n}\left(\mathbf{v}_{\mathbf{d}}^{\prime}-\mathbf{v}_{\mathbf{n}}^{\prime}\right)+\rho_{d}^{\prime} v_{d n}\left(\mathbf{v}_{\mathbf{d} \mathbf{0}}-\mathbf{v}_{\mathbf{n} \mathbf{0}}\right)+\rho_{n}^{\prime} \mathbf{g} \\
\frac{\partial \mathbf{B}^{\prime}}{\partial t}=\nabla \times\left(\mathbf{v}_{\mathbf{d}}^{\prime} \times \mathbf{B}_{\mathbf{0}}\right)+\nabla \times\left(\mathbf{v}_{\mathbf{d} \mathbf{0}} \times \mathbf{B}^{\prime}\right)
\end{gathered}
$$

Fourier analyzing the perturbations by taking the following form

$$
\Psi(x, y, z, t)=\Psi(y) \exp \left(\omega t+i k_{x} x+i k_{z} z\right)
$$

where $\Psi$ represents any of the perturbed field quantities of the considered dusty plasma, $\omega$ is the frequency, and $k_{x}$ and $k_{z}$ are the wavenumbers along $x$ - and $z$ - axes, we obtain

$$
-i k_{x} \frac{B_{0}^{2}}{4 \pi} \frac{\partial v_{d y}}{\partial y}-\left[\rho_{d 0}\left(\tilde{\omega}-v_{0} k_{z}\right)^{2}-k^{2} \frac{B_{0}^{2}}{4 \pi}+i \rho_{d 0} v_{d n}\left(\tilde{\omega}-v_{0} k_{z}\right)\right] v_{d x}+i \rho_{d 0} v_{d n}\left(\tilde{\omega}-v_{0} k_{z}\right) v_{n x}+k_{x} p_{c}\left(\tilde{\omega}-v_{0} k_{z}\right)=0
$$

$$
\begin{aligned}
& {\left[-i\left(\tilde{\omega}-v_{0} k_{z}\right)^{2} \rho_{d 0}+i \frac{B_{0}^{2}}{4 \pi} k_{z}^{2}+v_{d n}\left(\tilde{\omega}-v_{0} k_{z}\right) \rho_{d 0}-i g \frac{\partial \rho_{d 0}}{\partial y}\right] v_{d y}-i \frac{B_{0}^{2}}{4 \pi} \frac{\partial^{2} v_{d y}}{\partial y^{2}}+k_{x} \frac{B_{0}^{2}}{4 \pi} \frac{\partial v_{d x}}{\partial y}-v_{d n}\left(\tilde{\omega}-v_{0} k_{z}\right) \rho_{d 0} v_{n y}} \\
& +\left(\tilde{\omega}-v_{0} k_{z}\right) \frac{\partial p_{c}}{\partial y}=0
\end{aligned}
$$




$$
\begin{aligned}
& \rho_{d 0}\left(\tilde{\omega}-v_{0} k_{z}\right)\left[v_{d n}-i\left(\tilde{\omega}-v_{0} k_{z}\right)\right] \frac{\partial v_{d y}}{\partial y}+k_{x} \rho_{d 0}\left(\tilde{\omega}-v_{0} k_{z}\right)\left[\left(\tilde{\omega}-v_{0} k_{z}\right)+i v_{d n}\right] v_{d x}-\rho_{d 0} v_{d n} \\
& \left(\tilde{\omega}-v_{0} k_{z}\right) \frac{\partial v_{n y}}{\partial y}-v_{d n} k_{z} v_{0} \frac{\partial \rho_{d 0}}{\partial y} v_{d y}-i\left(\tilde{\omega}-v_{0} k_{z}\right) v_{d n} k_{x} \rho_{d 0} v_{n x}+\left(\tilde{\omega}-v_{0} k_{z}\right) k_{z}^{2} p_{c}=0 \\
& \left(-\rho_{n 0} \tilde{\omega}-i \rho_{d 0} v_{d n}\right) v_{n x}+i \rho_{d 0} v_{d n} v_{d x}+k_{x} p_{n}=0 \\
& \left(-i \tilde{\omega}^{2} \rho_{n 0}+\rho_{d 0} v_{d n} \tilde{\omega}-i g \frac{\partial \rho_{n 0}}{\partial y}\right) v_{n y}-\rho_{d 0} v_{d n} \tilde{\omega} v_{d y}+\tilde{\omega} \frac{\partial p_{n}}{\partial y}=0 \\
& \left.\quad-i \rho_{n 0} \tilde{\omega}\left(\tilde{\omega}-v_{0} k_{z}\right)+\rho_{d 0} v_{d n}\left(\tilde{\omega}-v_{0} k_{z}\right)\right] \frac{\partial v_{n y}}{\partial y}+\left[k_{x} \tilde{\omega}\left(\tilde{\omega}-v_{0} k_{z}\right) \rho_{n 0}+i k_{x} \rho_{d 0} v_{d n}\left(\tilde{\omega}-v_{0} k_{z}\right)\right] v_{n x} \\
& -\rho_{d 0} v_{d n}\left(\tilde{\omega}-v_{0} k_{z}\right) \frac{\partial v_{d y}}{\partial y}+v_{d n} k_{z} v_{0} \frac{\partial \rho_{d 0}}{\partial y} v_{d y}-i \rho_{d 0} k_{x} v_{d n}\left(\tilde{\omega}-v_{0} k_{z}\right) v_{d x}+k_{z}^{2}\left(\tilde{\omega}-v_{0} k_{z}\right) p_{n}=0
\end{aligned}
$$

where $k^{2}=k_{x}^{2}+k_{z}^{2}$ and $\tilde{\omega}=i \omega-k_{z} v_{n 0}$ is Doppler-shifted complex frequency.

We assume that surface perturbations $v_{d, n y}$ decay exponentially as $v_{d, n y}=v_{d, n y}(0) \exp \left( \pm k_{y} y\right)$ as $y \rightarrow \pm \infty$. We take $v_{d, n x}, v_{d, n z}, \frac{\partial v_{d, n y}}{\partial y}$ and $p_{d, n}$ are odd functions of $y$ for the surface perturbations. Hence, $\left[\tilde{\omega}^{2} \rho_{d 0} v_{d x}\right]=\tilde{\omega}^{2} \bar{\rho}_{d 0}\left[v_{d x}\right],\left[\tilde{\omega}^{2} \rho_{d 0} \partial v_{d y} / \partial y\right]=-2 k_{y} \tilde{\omega}^{2} \bar{\rho}_{d 0} v_{d y}(0)$, and $\int_{I}^{I I} \tilde{\omega} \partial \rho / \partial y d y=\tilde{\omega}\left[p_{c}\right]$, where bracket [] denotes the jump across the interface $(y=0)$ and an overline on physical quantity represents the mean value of that quantity as $\phi=1 / 2\left(\phi_{I}+\phi_{I I}\right)$. From equations (16) - (21), the coefficient determinant $C$ of $i v_{d y}(0),\left[v_{d x}\right], \tilde{\omega}\left[p_{c}\right], v_{n y}(0),\left[v_{n x}\right], \tilde{\omega}\left[p_{n}\right]$ is given by

$$
C=\left|\begin{array}{cccccc}
M & R & k_{x} & 0 & i \Omega_{1} & 0 \\
\zeta & k_{x} \frac{B_{0}^{2}}{4 \pi} & 1 & 0 & 0 & 0 \\
2 k_{y} D & k_{x} D & k_{z}^{2} & 2 k_{y} \Omega_{1} & -i k_{x} \Omega_{1} & 0 \\
0 & i \bar{\rho}_{d 0} v_{d n} & 0 & 0 & -A & k_{x} \\
0 & 0 & 0 & -i\left[\rho_{n 0}\right] g & 0 & \tilde{\omega} \\
-2 i k_{y} \Omega_{1} & -i k_{x} \Omega_{1} & 0 & 2 k_{y} F_{1} & k_{x} F_{2} & k_{z}^{2} \tilde{\omega}_{1}
\end{array}\right|
$$

where 


$$
\begin{aligned}
& \tilde{\omega}_{1}=\tilde{\omega}-k_{z} v_{0}, \quad \Omega=v_{d n} \tilde{\omega} \bar{\rho}_{d 0}, \quad \Omega_{1}=v_{d n} \tilde{\omega}_{1} \bar{\rho}_{d 0} \\
& A=-\tilde{\omega} \bar{\rho}_{n 0}-i \bar{\rho}_{d 0} v_{d n}, \quad \zeta=-g\left[\rho_{d 0}\right]+k_{y} \frac{B_{0}^{2}}{2 \pi} \\
& D=\tilde{\omega}_{1}^{2} \bar{\rho}_{n 0}+i \Omega_{1}, \quad M=k_{x} k_{y} \frac{B_{0}^{2}}{2 \pi}, \quad R=k^{2} \frac{B_{0}^{2}}{4 \pi}-D \\
& F_{1}=i \tilde{\omega} \tilde{\omega}_{1} \bar{\rho}_{n 0}-\Omega_{1}, \quad F_{2}=\tilde{\omega} \tilde{\omega}_{1} \bar{\rho}_{n 0}+i \Omega_{1}
\end{aligned}
$$

The determinant $C=0$ gives the following characteristic complex polynomial $\omega^{8}+\left(a_{1}+i b_{1}\right) \omega^{7}+\left(a_{2}+i b_{2}\right) \omega^{6}+\left(a_{3}+i b_{3}\right) \omega^{5}+\cdots \cdots+\left(a_{8}+i b_{8}\right)=0$ of eighth order in $\omega$. To discuss the instability of the system, we follow the Hurwitz criterion described by Hurwitz [14] and Giaretta [15], and construct the test sequence $h_{0}=1, h_{1}=a_{1} \cdots$.

$$
h_{r}=(-1)^{r(r-1) / 2}\left|\begin{array}{ccccccc}
a_{1} & a_{0} & 0 & 0 & 0 & \cdot & \cdot \\
-b_{2} & -b_{1} & a_{1} & a_{0} & 0 & \cdot & \cdot \\
a_{3} & a_{2} & b_{2} & b_{1} & a_{1} & \cdot & \cdot \\
-b_{4} & -b_{3} & a_{3} & a_{2} & -b_{2} & \cdot & \cdot \\
a_{5} & a_{4} & b_{4} & b_{3} & a_{3} & \cdot & \cdot \\
\cdot & \cdot & . & . & \cdot & \cdot & \cdot \\
\cdot & . & . & . & . & \cdot & \cdot \\
a_{2 r-1} & a_{2 r-2} & b_{2 r-2} & b_{2 r-3} & a_{2 r-3} & \cdot & \cdot
\end{array}\right|
$$

for the above complex polynomial. The condition for the instability is that any one of the $h_{i}{ }^{\prime} s$ be negative. It can be obtained from the third Hurwitz sub-determinant

$$
h_{2}=(-1)\left|\begin{array}{ccc}
a_{1} & 1 & 0 \\
-b_{2} & -b_{1} & a_{1} \\
a_{3} & a_{2} & b_{2}
\end{array}\right|<0
$$

Hence the instability condition derived from equation (24) is given by

$$
g\left(\rho_{I I}-\rho_{I}\right)>\frac{k_{y}}{k^{2}}\left(k_{z}^{2} \frac{B_{0}^{2}}{2 \pi}+\left(\rho_{I}+\rho_{I I}\right)\left(8 v_{d n}^{2}+\frac{1}{2} k_{z}^{2} v_{0}^{2}\right)\right)
$$

If we consider that there is no relative velocity $v_{0}$ i.e $v_{d 0}=v_{n 0}$ then the instability condition reduces to

$$
g\left(\rho_{I I}-\rho_{I}\right)>\frac{k_{y}}{k^{2}}\left(k_{z}^{2} \frac{B_{0}^{2}}{2 \pi}+8\left(\rho_{I}+\rho_{I I}\right) v_{d n}^{2}\right)
$$

This condition for instability is same to that condition (15) obtained by Birk [12]. It can be observed from condition (25) that stabilization occurs for modes with $k_{z}=0$ due to dust -neutral gas collisions only. The relative flow between dust and neutral gas tries to quench the instability, as one can see in case of no significant magnetic field the modes with wavenumber 


$$
k_{y}=\frac{k^{2} g\left(\rho_{I I}-\rho I\right)}{\left(\rho_{I}+\rho_{I I}\right)\left(8 v_{d n}^{2}+\frac{1}{2} k_{z}^{2} v_{0}^{2}\right)}
$$

are stabilized. If the neutral gas is homogeneous i.e. $\rho_{n I}=\rho_{n I I}=\rho_{n}$, the unstable modes grow in the dust fluid component and satisfy the criterion

$$
\begin{aligned}
& \left(\rho_{d I I}-\rho_{d I}\right)\left(\rho_{d I}+\rho_{d I I}+2 \rho_{n}\right) \rho_{n}^{3} g>\left(\rho_{d I}+\rho_{d I I}+2 \rho_{n}\right) \rho_{n}^{3} \frac{k_{y} k_{z}^{2}}{k^{2}} \frac{B_{0}^{2}}{\pi}+ \\
& {\left[\frac{1}{4}\left(\rho_{d I}+\rho_{d I I}\right)\left(\rho_{d I}+\rho_{d I I}+2 \rho_{n}\right)^{4} v_{d n}^{2}+2 k_{z}^{2} v_{0}^{2}\left(\rho_{d I}+\rho_{d I I}\right)^{2} \rho_{n}^{3}\right] \frac{k_{y}}{k^{2}}}
\end{aligned}
$$

In the absence of relative velocity $v_{0}$ the instability condition reduces to

$$
\begin{aligned}
& \left(\rho_{d I I}-\rho_{d I}\right)\left(\rho_{d I}+\rho_{d I I}+2 \rho_{n}\right) \rho_{n}^{3} g>\left(\rho_{d I}+\rho_{d I I}+2 \rho_{n}\right) \rho_{n}^{3} \frac{k_{y} k_{z}^{2}}{k^{2}} \frac{B_{0}^{2}}{\pi}+ \\
& {\left[\frac{1}{4}\left(\rho_{d I}+\rho_{d I I}\right)\left(\rho_{d I}+\rho_{d I I}+2 \rho_{n}\right)^{4} v_{d n}^{2}\right] \frac{k_{y}}{k^{2}}}
\end{aligned}
$$

If we simplify it, we obtain a similar condition to that condition (16) obtained by Birk [12] except a slight change in the constant coefficient of $\rho_{d I}^{5}$ and $\rho_{d I I}^{5}$. If we consider homogeneous dust component i.e. $\rho_{d I}=\rho_{d I I}=\rho_{d}$ and inhomogeneous neutral gas, the unstable growing modes satisfy the following condition

$$
\begin{aligned}
& {\left[\left(\rho_{n I I}^{2}-\rho_{n I}^{2}\right)\left(\rho_{n I}+\rho_{n I I}+2 \rho_{d}\right)\right] \rho_{d}^{2} g>\frac{1}{4}\left[\left(\rho_{n I}+\rho_{n I I}\right)^{3}\left(\rho_{n I}+\rho_{n I I}+2 \rho_{d}\right)\right] \times} \\
& \frac{k_{y} k_{z}^{2}}{k^{2}} \frac{B_{0}^{2}}{\pi}+\left[\left(\rho_{n I}+\rho_{n I I}+2 \rho_{d}\right)^{4} v_{d n}^{2}+2 k_{z}^{2} v_{0}^{2}\left(\rho_{n I}+\rho_{n I I}\right)^{3} \rho_{d}\right] \rho_{d} \frac{k_{y}}{k^{2}}
\end{aligned}
$$

In the absence of relative velocity $v_{0}$ this criterion reduces to

$$
\begin{aligned}
& {\left[\left(\rho_{n I I}^{2}-\rho_{n I}^{2}\right)\left(\rho_{n I}+\rho_{n I I}+2 \rho_{d}\right)\right] \rho_{d}^{2} g>\frac{1}{4}\left[\left(\rho_{n I}+\rho_{n I I}\right)^{3}\left(\rho_{n I}+\rho_{n I I}+2 \rho_{d}\right)\right] \times} \\
& \frac{k_{y} k_{z}^{2}}{k^{2}} \frac{B_{0}^{2}}{\pi}+\left[\left(\rho_{n I}+\rho_{n I I}+2 \rho_{d}\right)^{4} v_{d n}^{2}\right] \rho_{d} \frac{k_{y}}{k^{2}} v_{d n}^{2}
\end{aligned}
$$

which is similar to condition (18) obtained by Birk [12].

\section{CONCLUSIONS}

In this paper, we have studied the effect of flow on fluid dynamical instabilities in magnetized partially ionized dense dusty quasineutral plasma with dynamics governed by dust and neutral gas components. The electron dynamics is not considered as electrons have no significant influence on the overall behaviour of dusty plasma. The instability conditions have been derived for three different cases. Thus, the onset criteria for unstable modes obtained by Birk [12] have been modified in the presence of relative flow in dust and neutral gas. The relative flow between dust and neutral gas has stabilizing effect on the system. The results might be useful in many situations of astrophysical magnetized dusty plasmas namely comets and circumstellar dusty disks e.g. T-Tauri stars. 


\section{ACKNOWLEDGEMENTS}

One of us, N.K., is grateful to the IUCAA, Pune for the support and hospitality provided during my stay at IUCAA.

\section{REFERENCES}

[1] P.N.Foster, A.P.Boss, "Injection of Radioactive Nuclides from the Stellar Source that Triggered the Collapse of the Presolar Nebula", Astrophysical Journal, Vol. 489, pp. 346. 1997.

[2] J. Matsumoto, Y. Masada, "Two-dimensional Numerical Study for Rayleigh-Taylor and Richtmyer-Meshkov Instabilities in Relativistic Jets", Astrophysical Journal, Vol. 772, pp. L1, 2013.

[3] E. Ott, " Theory of Rayleigh-Taylor bubbles in the equatorial ionosphere", Journal of Geophysical Research, , Vol. 83, pp. $2066,1978$.

[4] S. L. Ossakow, S.T. Zalesak, B. E. McDonald, P. K. Chaturvedi, "Nonlinear equatorial spread F-Dependence on altitude of the F peak and bottomside background electron density gradient scale length", Journal of Geophysical Research, Vol. 84, pp. 17, 1979.

[5] D' Angelo "The Rayleigh-Taylor instability in dusty plasmas", Planetary and Space Science, Vol. 41, pp. 469, 1993.

[6] R. K. Varma, P.K. Shukla, “A new dust-dynamics-induced interchange instability in dusty plasmas”, Physics Letters A, Vol. 169, pp. $342,1995$.

[7] S.Chandrasekhar, "Hydrodynamic and Hydromagnetic Stabilty", Clarendon Press. Oxford, 1961.

[8] G. K. Goertz, “ Dusty plasmas in the solar system”, Reviews of Geophysics, Vol. 27, pp. 271, 1989.

[9] G.T. Birk, H. Wiechen, H. Lesch, P. P. Kronberg, "The role of Kelvin-Helmholtz modes in superwinds of primeval galaxies for the magnetization of the intergalactic medium” Astronomy \& Astrophysics, Vol. 353, pp. 108, 2000.

[10] H. Wiechen, G.T. Birk, H. Lesch, "The origin of primeval magnetic fields: Self-consistent simulation of magnetic field generation and amplification", Physics of Plasmas, Vol. 7, pp. 701, 2000.

[11] H. Kamaya, R. Nishi, "Fluid Dynamical Instabilities in a Partially Ionized Flow”, Astrophysical Journal Vol. 534, pp. 309, 2000.

[12] G.T. Birk," The onset of Rayleigh-Taylor instabilities in magnetized partially ionized dense dusty plasmas", Physics of Plasmas, Vol. 9, pp. 745, 2002.

[13] G.T. Birk, H. Wiechen, "Shear flow instabilities in magnetized partially ionized dense dusty plasmas", Physics of Plasmas, Vol. 9, pp. 964, 2002.

[14] A. Hurwitz, "Ueber die Bedingungen, unter welchen eine Gleichung nur Wurzeln mit negativen reellen Theilen besitzt", Mathematische Annalen, Vol. 46, pp. 273, 1865.

[15] D.L. Giaretta, “Polynomial dispersion relations" Astronomy \& Astrophysics, Vol. 75, pp. 273, 1979. 